\title{
Effect of local terrain in neutrino propagation based on Simulation with topographic data
}

\author{
T.C. Liu $^{1}$ \\ ${ }^{1}$ LeCosPA center, Department of Physics, National Taiwan University \\ 1, Roosevelt Rd., Taipei, Taiwan, 10617, ROC \\ E-mail: tcliu@ntu.edu.tw
}

\section{M.H. Huang ${ }^{1,2}$}

${ }^{2}$ Department of Energy Engineering, National United University

2 Lienda Rd., Miaoli county, Taiwan, 36063, ROC

E-mail: mahuang@neutrinou.edu.tw

\section{C.J. Chi ${ }^{3}$, Chia-Hao $\mathbf{W u}^{3}$}

${ }^{3}$ Institute of Physic, National Chiao-Tung University

1001 University Road, Hsinchu 30010, Taiwan, ROC

\begin{abstract}
Extremely high energy neutrinos are attenuated by the materials surrounding the neutrino detector. Topography data can provides spatial distribution of material and become an essential factor in high energy neutrino experiment, especially for the ultra high energy neutrinos. This study introduced the Antarctica topography data, including composite layers of rock, ice, and water, to investigate the topography effect neutrino events from near horizon. Azimuthal asymmetry were observed due different depth of materials along the neutrino trajectories from different direction. This model can be applied to different neutrino experiment (balloon borne detector, ground-level detector, and underground detector) to produce realistic estimation of the neutrino induced events under different geometry of detectors.
\end{abstract}

The 34th International Cosmic Ray Conference

30 July- 6 August, 2015

The Hague, The Netherlands

${ }^{1}$ Speaker 


\section{Introduction}

The interaction cross section of very high energy neutrino interacting with nucleon increase as $\mathrm{E}^{0.37}$ at energy higher than $10^{15} \mathrm{eV}(1 \mathrm{PeV}$ ) [1]. At neutrino energy above $\mathrm{PeV}$, the cross-section is large enough that the Earth become opaque. For ultra high energy neutrinos, (UHE-v) at around $10^{18} \mathrm{eV}(\mathrm{EeV})$, neutrinos can be detected only from shallow angles below horizon. Near the horizon, the local terrain may be quite different from different angles. Material types, density, and their distribution are crucial to determine interaction depth, which the interaction probability of neutrinos depends on. The large changes in material distribution create large variations in the modulation of neutrino fluxes.

For a neutrino detector targeting at UHE-v, the local terrain must be taken into considerations besides the intrinsic Earth model. In our previous simulation package, SHINIE, the Earth is treated as a sphere and the local materials must be supplied by users. The default model is a layer of constant depth made from several materials such as water, ice, and standard rock. Both depth and materials can be defined in the input card for simulation. This simplified model provides quick and effective modeling of neutrino fluxes along zenith angles. However, the azimuthal distribution is symmetric. A realistic local terrain model can be added by users.

This study upgrades previous model with two important features, the elliptical shape of Earth and digital elevation model (DEM) of Antarctica. This DEM, called Antarctica Bedmap2, includes boundaries between sea, rock, and ice. These data are used to determined the material types, which then density and energy loss depends on.

This paper reports those two upgrades in section 2. Section 3 discusses simulation for a balloon borne / underground detector such as ANITA / ARA. Section 4 presents some typical distributions of lepton fluxes induced by cosmogenic neutrinos.

\section{Realistic Model of Earth and Local Terrain}

Based on the standard model and CTEQ3 parton distribution model, Gandhi et al. drive the cross-section of neutrino and nucleon interactions [1]. At neutrino energy $10^{17} \mathrm{eV}, v-N$ crosssection is $6.3 \times 10^{-33} \mathrm{~cm}^{2}$, the interaction length in standard rock of density $2.65 \mathrm{~g} / \mathrm{cm}^{2}$ is 1000 $\mathrm{km}, \sim 1 / 6$ of the radius of the Earth, or equivalent to the cord length of Earth for a neutrino coming from 4.5 degree below horizon. The interaction length is shorter at higher energy because of the $v-N$ cross-section increases as $E^{0.363}$, where $\mathrm{E}$ is energy of neutrino energy. UHE Neutrino telescope can detect neutrino induced events from direction near horizon, where local terrain may cause large fluctuation in the total depths, which depends on the materials and length of materials that neutrino of leptons traveled. Therefore a detail modeling of local terrain is crucial for precise measurement and interpretation of neutrino energy.

In a previous model, SHINIE, of neutrinos interacting inside the Earth, we use a spherical Earth with radius $6371.2 \mathrm{~km}$ [2, 3]. The material density inside the Earth is modeled by a density profile, $\rho(r)$, where $r$ is radius in spherical Earth model. The total depth is a integration of $\rho(r)$ along the path that neutrino transverse. This model provides fair representation of neutrino at $\mathrm{PeV}$ to EeV, where neutrino interaction length is of the order of several thousands $\mathrm{km}$. The local terrain was used when neutrino is close to the neutrino telescope. Users must supply their digital 
elevation map (DEM) within $100 \mathrm{~km}$ around neutrino telescope. $100 \mathrm{~km}$ is approximately distance of visible horizon.

In this study, we update two important geometry, shown as figure 1. The spherical Earth is replaced with a ellipsoid, which is known as WGS84 model, in which Earth has semi-major length of $6378.137 \mathrm{~km}$ and semi-minor length of $6356.752 \mathrm{~km}$. The internal structure such as core, mantle, and crust and assumed have the same eccentricity. The density profile $\rho(r)$ is replaced with a effective radius $r^{\prime}$ depends on its position relative to the center of the Earth. This improvement is more important for neutrino energy near $\mathrm{PeV}$, where the neutrino attenuation is sensitive to the zenith angle of neutrino direction.

To be valid for energy higher than EeV, and can be applied to experiment on Antarctica, we use Bedmap2 for DEM. Bedmap2 use polar projection grid, which covers the area from $60^{\circ}$ $\mathrm{S}$ to $90^{\circ} \mathrm{S}$. The WGS84 Earth model and Bedmap2 merge at the edges of DEM, and the rest of Earth is assumed as featureless.
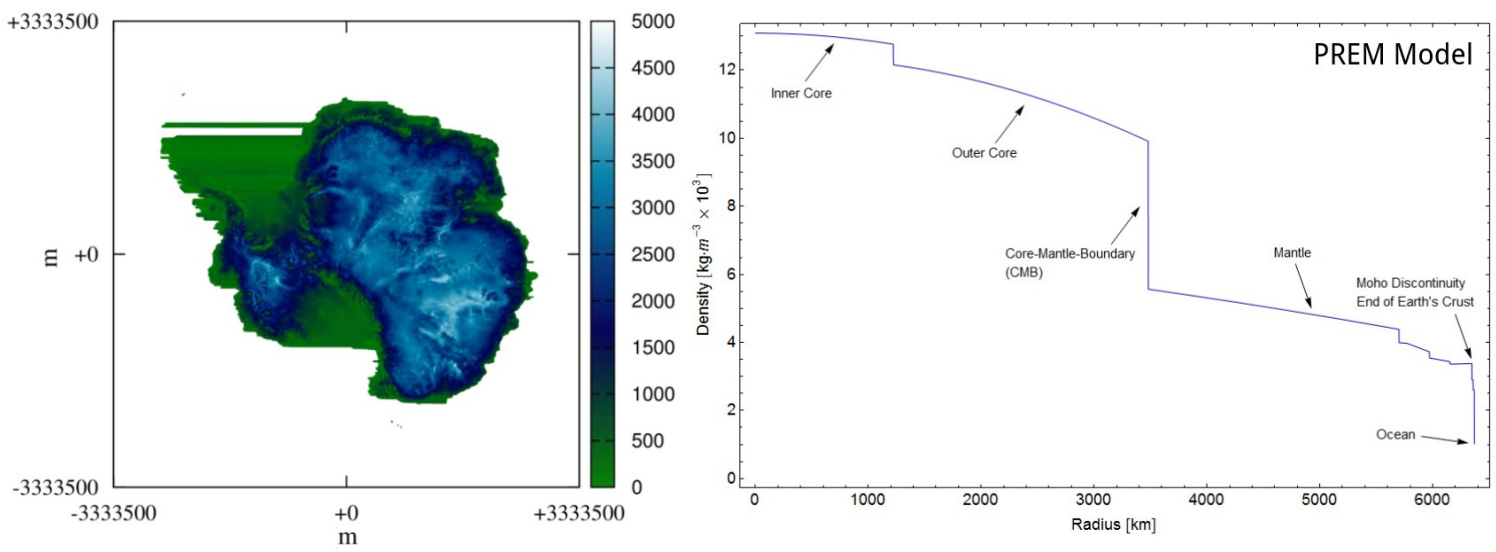

Figure 1: Left panel: The thickness of the Ice of Antarctica(Bedmap2 data). Right panel: The mantle density model. In this work, SHINIE using the ellipsoid Geometry with PREM Model [2, 3, 4].

\section{Slant depth of neutrinos}

To demonstrate the effect of local terrains, we calculate the slant depth for two types of neutrino detectors. The First one is a underground detector array at the South pole [5]. The detector is $200 \mathrm{~m}$ below the icy ground level. Figure 2 shows the slant depth of neutrino trajectory is dependent on the neutrino direction, zenith angle. The local terrain causes the fluctuation of the total depths along the neutrino trajectory. Zenith angle $\theta$ equals $0^{\circ}$ means this event comes from the zenith, and $\theta=180^{\circ}$ means this events comes from nadir. Or, $\theta<90^{\circ}$ are the down-going events and $\theta>90^{\circ}$ are the up-going events. 


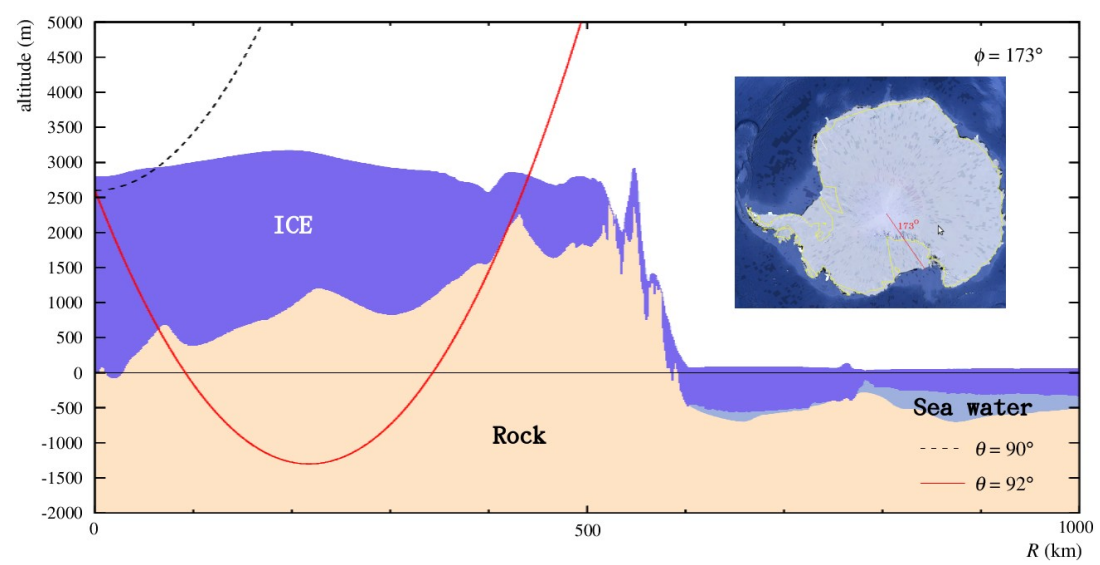

Figure 2: The trajectory of propagation path along $173^{\circ} \mathrm{W}$ start from $200 \mathrm{~m}$ depth ice of south pole with different zenith angle, $90^{\circ} \& 92^{\circ}$. The interaction length of neutrino with energy $10^{17} \mathrm{eV}$ is about $1000 \mathrm{~km}$. The local terrain causes the fluctuation of the total depths along the neutrino trajectory.

Figure 3 is an enlarge view of sated depth for $\theta$ at $89^{\circ}$ to $95^{\circ}$. The difference start to shown up in the zoom-in figure. Three extra lines correspond to the maximum, mean, and minimum of depths when scan the different azimuth angles while keep the same zenith angle. The difference between the maximum and minimum could be as large as 1.8 times.

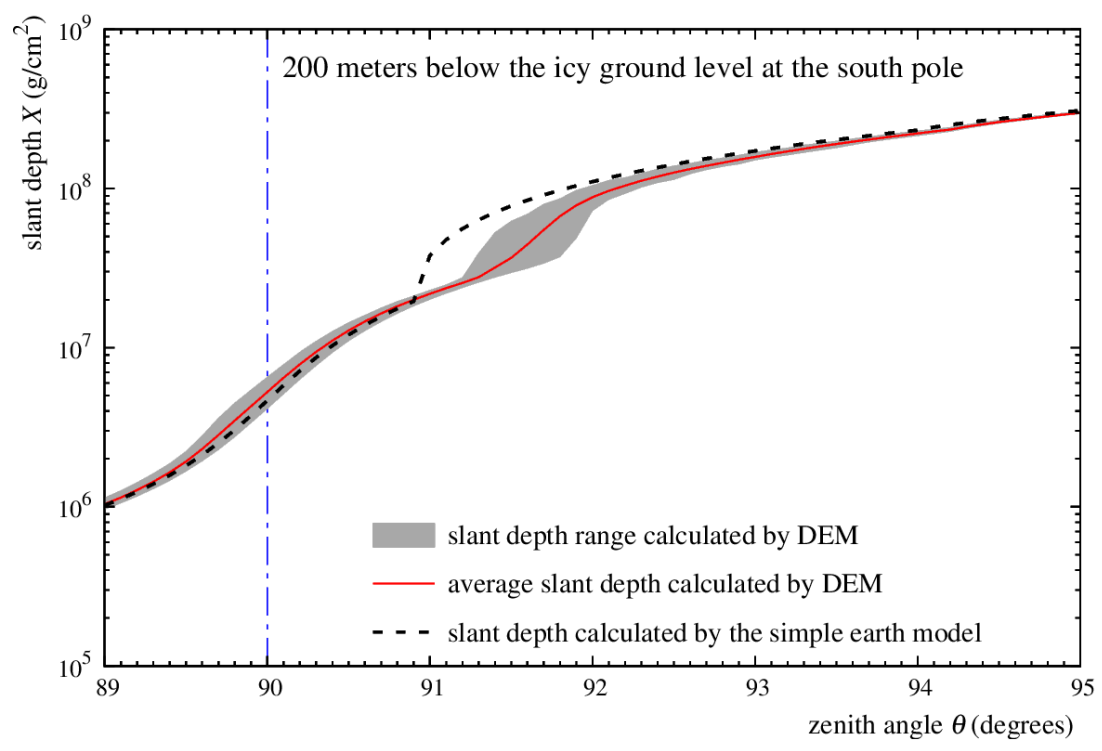

Figure 3: Slant depth of neutrino path at different zenith angle for a underground detector at the South pole. The dash line is the simple Earth model without local terrain (Antarctica). The red solid line is Earth plus the Antarctica topological data, Bedmap2. The gray area denote the fluctuation of slant depth that scanning the different azimuth angle while keep the same zenith angle.

The other type of detector is a balloon borne experiment, such as ANITA [6], which use radio antenna array to scan the vast area of ice surface of the Antarctica. The down going events 
pass through the atmosphere only, most events are up-going events. The slanted depth distribution is plotted in Figure 4, with convention similar to that in figure 2.

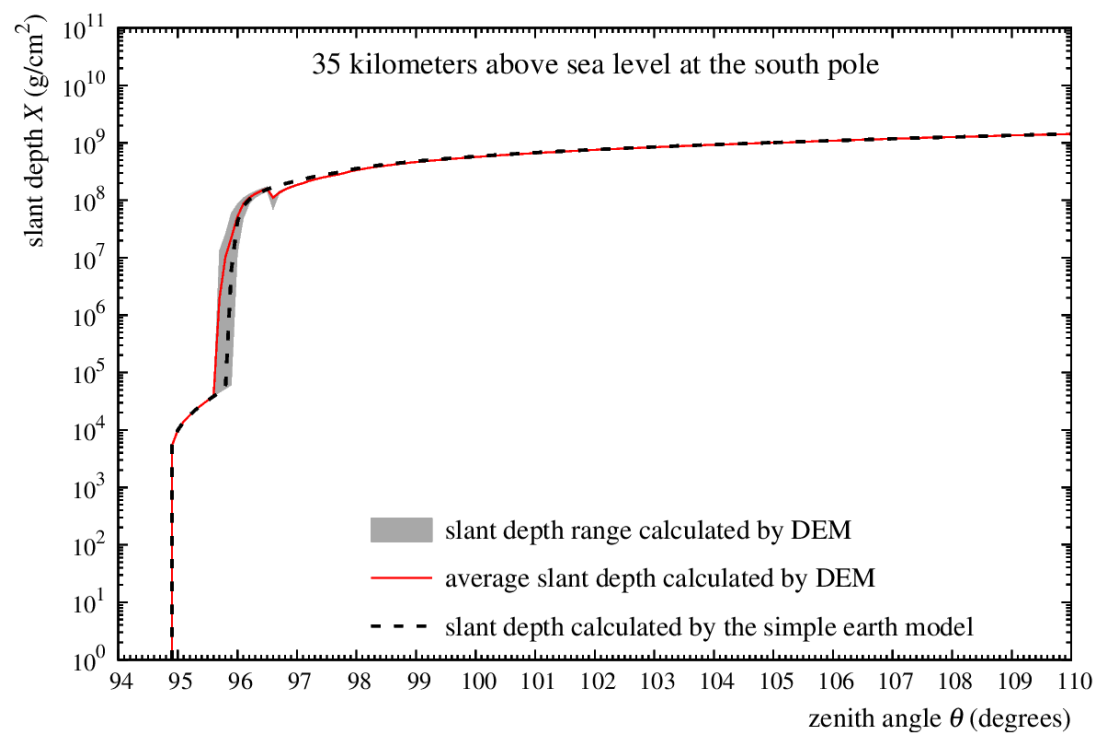

Figure 4: Slant depth of neutrino path at different zenith angle for a balloon-borne detector[6]. The elevation of balloon-borne detector is set at $35 \mathrm{~km}$ high sky and fixed at the South pole center.

The difference in figure 4 may looks small, but if we fix at one zenith angle and look at azimuth distribution, then the azimuth asymmetry become apparent. Figure 5 shows the azimuthal distribution of slant depth at zenith angle $\theta=92^{\circ}$.

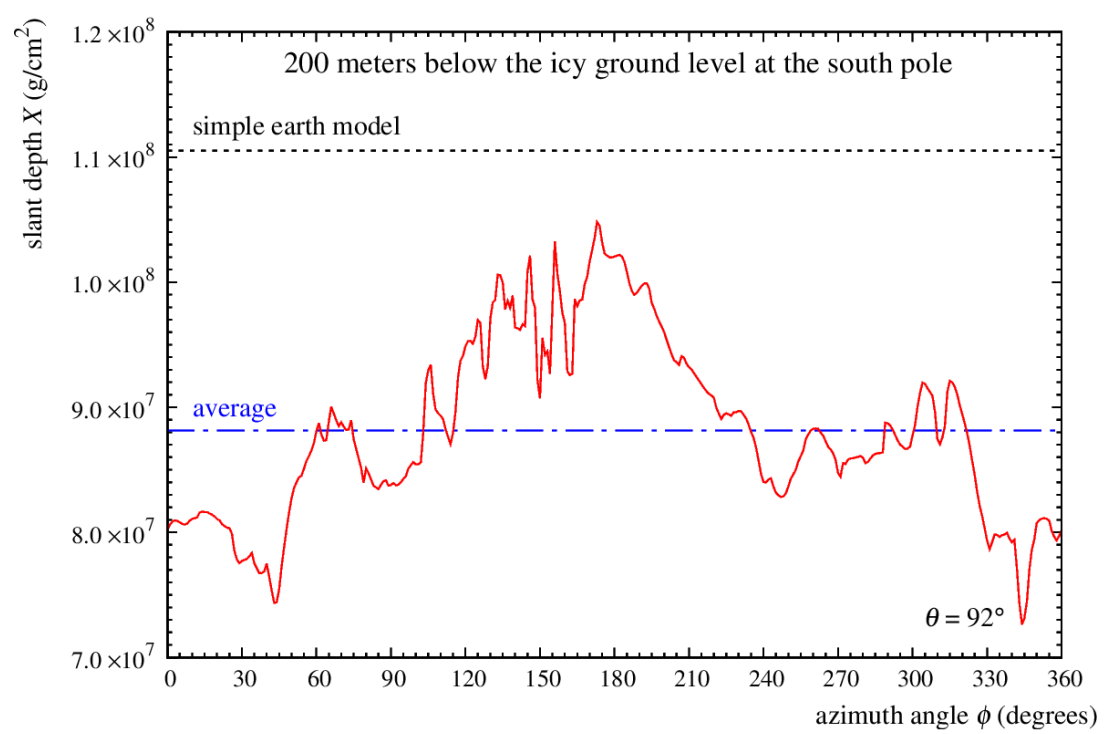

Figure 5: Azimuth distribution of slant depth for a 200m depth underground detector at the South pole and looking at zenith angle $\theta=92^{\circ}$. 


\section{Conclusion}

We had upgraded neutrino interaction model with WGS84 Earth model and add Antarctica topological data model Bedmap2. These updates produced fluctuations of slant depth and therefore will produced fluctuation in the neutrino induced events fluxes, which is topics for on-going study and will be published in near future.

\section{References}

[1] R. Gandhi, et al., (1998) Ultra-high-energy neutrino interactions, Phys. Rev. D 58, 093009

[2] M.A. Huang, (2008), SHINIE: Simulation of High Energy Neutrino Interacting with the Earth, proceeding of ISVHECRI-2006, Weihai, China, (Aug., 2006), Nucl. Phys. B (proc. supl.), 175-176, 472-475; DOI: 10.1016/j.nuclphysbps.2007.11.055

[3] T.C. Liu, M.A. Huang, C.H. Iong, Guey-Lin Lin, (2009) Lepton fluxes from astrophysical neutrinos interacting inside the Earth, Proceedings of the 31st ICRC, Lodz, 2009, \#1082

[4] A. M. Dziewonski, D. L. Anderson, Physics of the Earth and Planetary Interiors 25 (4): 297-356.

[5] P. Allison, et al., for ARA collaboration, (2012), Design and Initial Performance of the Askaryan Radio Array Prototype EeV Neutrino Detector at the South Pole, Astropart. Phys., 35:457-477;

[6] ANITA collaboration, A.G. Vieregg, et al., (2011) The first limits on the ultra high energy neutrino flux from gamma ray bursts, Astrophys. J. 736: 50 